CUBO A Mathematical Journal

Vol.12, $N^{\mathrm{O}}$ 02, (1-17). June 2010

\title{
Existence Results for Semilinear Differential Evolution Equations with Impulses and Delay
}

\author{
NADJET ABADA \\ Département de Mathématiques, \\ Université Mentouri Constantine, \\ email: n65abada@yahoo.fr \\ MOUFFAK BENCHOHRA ${ }^{1}$ \\ Laboratoire de Mathématiques, \\ Université de Sidi Bel Abbès, \\ BP 89, 22000 Sidi Bel Abbès, Algérie. \\ email: benchohra@univ-sba.dz \\ and \\ HADDA HAMMOUCHE \\ Département de Mathematiques, \\ Université Kasdi Merbah Ouargla, \\ email: h.hammouche@yahoo.fr
}

\begin{abstract}
In this paper, we establish sufficient conditions for the existence of mild and extremal solutions for some densely defined impulsive functional differential equations in separable Banach spaces with local and nonlocal conditions. We shall rely for the existence of mild solutions on a fixed point theorem due to Burton and Kirk for the sum of completely continuous and contraction operators, and for the existence of extremal solutions on Dhage's fixed point theorem.
\end{abstract}

\footnotetext{
${ }^{1}$ Corresponding author
} 


\section{RESUMEN}

En este artículo establecemos condiciones suficientes para la existencia de soluciones suaves y extremas para algunas ecuaciones diferenciales funcionales impulsivas densamente definidas en espacios de Banach separables con condiciones locales y no locales. Para la existencia de soluciones suaves usaremos un teorema de punto fijo debido a Burton y Kirk para la suma de un operador completamente continuo y otro contractivo; para la existencia de soluciones extremas usaremos el teorema de punto fijo de Dhage.

Key words and phrases: Densely defined operator, impulsive functional differential equations, fixed point, semigroups, mild solutions, extremal mild solutions, nonlocal condition, separable Banach space.

AMS (MOS) Subj. Class.: 34A37, 34K30, 34K35, 34K45.

\section{Introduction}

In this paper, we establish sufficient conditions for the existence of mild and extremal mild solutions of first order impulsive functional equations in a separable Banach space $(E$. |.|) of the form:

$$
\begin{gathered}
y^{\prime}(t)-A y(t)=f\left(t, y_{t}\right), \text { a.e. } t \in J=[0, b], t \neq t_{k}, k=1, \ldots, m \\
\left.\Delta y\right|_{t=t_{k}}=I_{k}\left(y\left(t_{k}^{-}\right)\right), k=1, \ldots, m \\
y(t)=\phi(t), t \in[-r, 0],
\end{gathered}
$$

where $f: J \times D \rightarrow E$ is a given function, $D=\{\psi:[-r, 0] \rightarrow E, \psi$ is continuous every where except for a finite number of points $s$ at which $\psi\left(s^{-}\right), \psi\left(s^{+}\right)$exist and $\left.\psi\left(s^{-}\right)=\psi(s)\right\}, \phi \in D, 0<r<$ $\infty, 0=t_{0}<t_{1}<\ldots<t_{m}<t_{m+1}=b, I_{k} \in C(E, E), k=1,2, \ldots, m, A: D(A) \subset E \rightarrow E$ is the infinitesimal generator of a $C_{0}$-semigroup $T(t), t \geq 0$, and $E$ a real separable Banach space with norm $|$.$| .$

We denote by $y_{t}$ the element of $D$ defined by

$$
y_{t}(\theta)=y(t+\theta), \quad \theta \in[-r, 0]
$$

Here $y_{t}(\cdot)$ represents the history of the state from $t-r$, up to the present time $t$.

Functional differential equations arise in many areas of applied mathematics and such equations have received much attention in recent years. A good guide to the literature for functional differential equations is the books by Hale [18] and Hale and Verduyn Lunel [19] and Kolmanovskii and Myshkis [23] and the references therein.

Impulsive differential equations have become important in recent years as mathematical models of phenomena in both the physical and social sciences. There has a significant development in impulsive theory especially in the area of impulsive differential equations with fixed moments; see for instance the monoghraphs by Bainov and Simeonov [5], Benchohra et al [6], Lakshmikantham et al [24], and Samoilenko and Perestyuk [29]. In the case where the impulses are absent (i.e $I_{k}=0, k=1, \ldots, m$ ) and $F$ is a single or multivalued map and $A$ is a densely defined linear operator generating a $C_{0}$-semigroup 
of bounded linear operators the problem (1)-(3) has been investigated on compact intervals in, for instance, the monographs by Ahmed [1], Hu and Papageorgiou [21], Kamenskii et al [22] and Wu [30] and the papers of Benchohra and Ntouyas [7, 8, 9].

During the last decades problems of the form (1)-(3) have received much attention. Some existence results were given in the monograph Ahmed [2] and Benchohra et al [6] and the papers by Ahmed [3, 4], Cardinali and Rubbioni [15], Liu [25] and Rogovchenko [27, 28], and the references therein.

The plan of this paper is as follows: in Section 2, we will recall briefly some basic definitions and preliminary facts which will be used throughout the following sections. In Section 3, we prove existence of mild solutions for problem (1)-(3). Our approach will be based for the existence of mild solutions, on a fixed point theorem of Burton and Kirk [10] for the sum of a contraction map and a completely continuous map. In Section 4, we shall prove the existence of extremal solutions of the problem (1)-(3), and our approach here is based on the concept of upper and lower solutions combined with a fixed point theorem on ordered Banach spaces established recently by Dhage [16]. In Section 5 we study the impulsive functional differential equations with non-local initial conditions, of the form

$$
\begin{gathered}
y^{\prime}(t)-A y(t)=f\left(t, y_{t}\right), \quad \text { a.e. } t \in J=[0, b], t \neq t_{k}, k=1, \ldots, m \\
\left.\Delta y\right|_{t=t_{k}}=I_{k}\left(y\left(t_{k}^{-}\right)\right), k=1, \ldots, m \\
y(t)+h_{t}(y)=\phi(t), t \in[-r, 0],
\end{gathered}
$$

where $h_{t}: P C([-r, b], E) \rightarrow E$ is given function (see Section 2 for the definition of $P C([-r, b], E)$ ). The non-local condition can be applied in physics with better effect than the classical initial condition $y(0)=y_{0}$. For example, $h_{t}(y)$ may be given by

$$
h_{t}(y)=\sum_{i=1}^{p} c_{i} y\left(t_{i}+t\right), \quad t \in[-r, 0]
$$

where $c_{i}, i=1, \ldots, p$, are given constants and $0<t_{1}<\ldots<t_{p} \leq b$. At time $t=0$, we have

$$
h_{0}(y)=\sum_{i=1}^{p} c_{i} y\left(t_{i}\right)
$$

Non-local conditions were initiated by Byszewski [11] (see also [12, 13, 14]) in which we refer for motivation and other references. Finally, Section 6 is devoted to an example illustrating the abstract theory considered in the previous sections.

\section{Preliminaries}

In this section, we introduce notations and definitions, preliminaries facts which are used throughout this paper. For $\psi \in D$ the norm of $\psi$ is defined by

$$
\|\psi\|_{D}=\sup \{|\psi(\theta)|: \theta \in[-r, 0]\},
$$

and $B(E)$ denotes the Banach space of bounded linear operators from $E$ into $E$, with norm

$$
\|N\|_{B(E)}=\sup \{|N(y)|:|y|=1\} .
$$


$L^{1}(J, E)$ denotes the Banach space of measurable functions $y: J \longrightarrow E$ which are Bochner integrable normed by

$$
\|y\|_{L^{1}}=\int_{0}^{b}|y(t)| d t
$$

It is well known that the operator $A$ generates a $\left(C_{0}\right)$ semigroup if $A$ satisfies:

(i) $\overline{D(A)}=E$.

(ii) The Hille-Yosida condition, that is, there exists $M \geq 0$ and $\omega \in \mathbb{R}$ such that $(\omega, \infty) \subset \rho(A)$ and

$$
\sup \left\{(\lambda-\omega)^{n}\left|(\lambda I-A)^{-n}\right|: \lambda, n \in \mathbb{N}\right\} \leq M
$$

where $\rho(A)$ is the resolvent set of $A$ and $I$ is the identity operator in $E$.

For more details on semigroup theory we refer the interested reader to the books of Ahmed [1] and Pazy [26].

In order to define a mild solution of problems (1)-(3) and (4)-(6), we shall consider the space $P C([-r, b], E)=\left\{y:[-r, b] \rightarrow E: y(t)\right.$ is continuous everywhere except for some $t_{k}$ at which $y\left(t_{k}^{-}\right)$ and $y\left(t_{k}^{+}\right), k=1,2, \ldots, m$ exist and $\left.y\left(t_{k}^{-}\right)=y\left(t_{k}\right)\right\}$.

Obviously, for any $t \in[0, b]$ and $y \in P C([-r, b], E)$, we have $y_{t} \in D$ and $P C([-r, b], E)$ is a Banach space with the norm

$$
\|y\|=\sup \{|y(t)|: t \in[-r, b]\} .
$$

Definition 2.1. A map $f: J \times D \rightarrow E$ is said to be Carathéodory if

(i) the function $t \longmapsto f(t, x)$ is measurable for each $x \in D$;

(ii) the function $x \longmapsto f(t, x)$ is continuous for almost all $t \in J$.

\section{Existence of Mild Solutions}

In this section, we give our main existence result for problem (1)-(3). Before stating and proving this result, we give the definition of the mild solution.

Definition 3.1. A function $y \in P C([-r, b], E)$ is said to be a mild solution of problem (1)-(3) if $y(t)=\phi(t), t \in[-r, 0]$, and $y$ is a solution of impulsive integral equation

$$
y(t)=T(t) \phi(0)+\int_{0}^{t} T(t-s) f\left(t, y_{s}\right) d s+\sum_{0<t_{k}<t} T\left(t-t_{k}\right) I_{k}\left(y\left(t_{k}^{-}\right)\right), \quad t \in J .
$$

Our main result in this section is based upon the following fixed point theorem due to Burton and Kirk [10].

Theorem 3.1. Let $X$ be a Banach space, and $\mathcal{A}, \mathcal{B}: X \rightarrow X$ two operators satisfying:

(i) $\mathcal{A}$ is a contraction, and 
(ii) $\mathcal{B}$ is completely continuous.

Then either

(a) the operator equation $y=\mathcal{A}(y)+\mathcal{B}(y)$ has a solution, or

(b) the set $\mathcal{E}=\left\{u \in X: \lambda \mathcal{A}\left(\frac{u}{\lambda}\right)+\lambda \mathcal{B}(u)=u, \lambda \in(0,1)\right\}$ is unbounded.

Let us introduce the following hypotheses:

(H1) $A: D(A) \subset E \rightarrow E$ is the infinitesimal generator of a $C_{0}$-semigroup $\{T(t)\}, t \in J$ which is compact for $t>0$ in the Banach space $E$. Let $M=\sup \left\{\|T(t)\|_{B(E)}: t \in J\right\}$;

(H2) There exist constants $d_{k}>0, k=1, \ldots, m$ with $M \sum_{k=1}^{m} d_{k}<1$ such that

$$
\left|I_{k}(y)-I_{k}(x)\right| \leq d_{k}|y-x| \text { for each } y, x \in E
$$

(H3) The function $f: J \times D \rightarrow E$ is Carathéodory;

(H4) There exists a function $p \in L^{1}\left(J, \mathbb{R}_{+}\right)$and a continuous nondecreasing function $\psi:[0, \infty) \rightarrow$ $(0, \infty)$ such that

$$
|f(t, x)| \leq p(t) \psi\left(\|x\|_{D}\right), \quad \text { a.e. } t \in J, \quad \text { for all } x \in D
$$

with

$$
\int_{D_{o}}^{\infty} \frac{d s}{\psi(s)}>D_{1}\|p\|_{L^{1}}
$$

where

$$
D_{0}=\frac{M\left(\|\phi\|+\sum_{k=1}^{m}\left|I_{k}(0)\right|\right)}{1-M \sum_{k=1}^{m} d_{k}}, \quad D_{1}=\frac{M}{1-M \sum_{k=1}^{m} d_{k}} .
$$

Theorem 3.2. Assume that (H1)-(H4) hold. Then the problem (1)-(3) has at least one mild solution on $[-r, b]$.

Proof. Transform the problem (1)-(3) into a fixed point problem. Consider the two operators:

$$
\mathcal{A}, \mathcal{B}: P C([-r, b], E) \rightarrow P C([-r, b], E)
$$

defined by

$$
\mathcal{A}(y)(t):= \begin{cases}0, & \text { if } t \in[-r, 0] ; \\ \sum_{0<t_{k}<t} T\left(t-t_{k}\right) I_{k}\left(y\left(t_{k}^{-}\right)\right), & \text {if } t \in J,\end{cases}
$$

and 


$$
\mathcal{B}(y)(t):= \begin{cases}\phi(t), & \text { if } t \in[-r, 0] ; \\ T(t) \phi(0)+\int_{0}^{t} T(t-s) f\left(s, y_{s}\right) d s, & \text { if } t \in J .\end{cases}
$$

Then the problem of finding the solution of problem (1)-(3) is reduced to finding the solution of the operator equation $\mathcal{A}(y)(t)+\mathcal{B}(y)(t)=y(t), t \in[-r, b]$. We shall show that the operators $\mathcal{A}$ and $\mathcal{B}$ satisfy all the conditions of Theorem 3.1. For better readability, we break the proof into a sequence of steps.

Step 1: $\mathcal{B}$ is continuous.

Let $\left\{y_{n}\right\}$ be a sequence such that $y_{n} \rightarrow y$ in $P C([-r, b], E)$. Then for $t \in J$

$$
\begin{aligned}
\left|\mathcal{B}\left(y_{n}\right)(t)-\mathcal{B}(y)(t)\right| & =\left|\int_{0}^{t} T(t-s)\left[f\left(s, y_{n_{s}}\right)-f\left(s, y_{s}\right)\right] d s\right| \\
& \leq M \int_{0}^{b}\left|f\left(s, y_{n_{s}}\right)-f\left(s, y_{s}\right)\right| d s .
\end{aligned}
$$

Since $f(s, \cdot)$ is continuous for a.e. $s \in J$, we have by the Lebesgue dominated convergence theorem

$$
\left|\mathcal{B}\left(y_{n}\right)(t)-\mathcal{B}(y)(t)\right| \rightarrow 0 \text { as } n \rightarrow \infty \text {. }
$$

Thus $\mathcal{B}$ is continuous.

Step 2: $\mathcal{B}$ maps bounded sets into bounded sets in $P C([-r, b], E)$.

It is enough to show that for any $q>0$ there exists a positive constant $l$ such that for each $y \in B_{q}=\{y \in P C([-r, b], E):\|y\| \leq q\}$ we have $\|\mathcal{B}(y)\| \leq l$. So choose $y \in B_{q}$, then we have for each $t \in J$,

$$
\begin{aligned}
|\mathcal{B}(y)(t)| & =\left|T(t) \phi(0)+\int_{0}^{t} T(t-s) f\left(s, y_{s}\right) d s\right| \\
& \leq M|\phi(0)|+M \psi(q) \int_{0}^{b} p(s) d s ;
\end{aligned}
$$

Then we have

$$
\|\mathcal{B}(y)\| \leq M\|\phi\|+M \psi(q)\|p\|_{L^{1}}:=l .
$$

Step 3: $\mathcal{B}$ maps bounded sets into equicontinuous sets of $P C([-r, b], E)$.

We consider $B_{q}$ as in step 2 and let $\tau_{1}, \tau_{2} \in J \backslash\left\{t_{1}, \ldots, t_{m}\right\}, \tau_{1}<\tau_{2}$. Thus if $\epsilon>0$ and $\epsilon \leq \tau_{1}<\tau_{2}$ we have 


$$
\begin{aligned}
\left|\mathcal{B}(y)\left(\tau_{2}\right)-\mathcal{B}(y)\left(\tau_{1}\right)\right| \leq & \left|T\left(\tau_{2}\right) \phi(0)-T\left(\tau_{1}\right) \phi(0)\right| \\
& +\psi(q) \int_{0}^{\tau_{1}-\epsilon}\left\|T\left(\tau_{2}-s\right)-T\left(\tau_{1}-s\right)\right\|_{B(E)} p(s) d s \\
& +\psi(q) \int_{\tau_{1}-\epsilon}^{\tau_{1}}\left\|T\left(\tau_{2}-s\right)-T\left(\tau_{1}-s\right)\right\|_{B(E)} p(s) d s \\
& +\psi(q) \int_{\tau_{1}}^{\tau_{2}}\left\|T\left(\tau_{2}-s\right)\right\|_{B(E)} p(s) d s .
\end{aligned}
$$

As $\tau_{1} \rightarrow \tau_{2}$ and $\epsilon$ become sufficiently small, the right-hand side of the above inequality tends to zero, since $T(t)$ is a strongly continuous operator and the compactness of $T(t)$ for $t>0$ implies the continuity in the uniform operator topology $([1,26])$. This proves the equicontinuity for the case where $t \neq t_{i}, k=1,2, \ldots, m+1$. It remains to examine the equicontinuity at $t=t_{i}$.

First we prove equicontinuity at $t=t_{i}^{-}$. Fix $\delta_{1}>0$ such that $\left\{t_{k}: k \neq i\right\} \cap\left[t_{i}-\delta_{1}, t_{i}+\delta_{1}\right]=\emptyset$. For $0<h<\delta_{1}$ we have

$$
\begin{aligned}
\left|\mathcal{B}(y)\left(t_{i}-h\right)-\mathcal{B}(y)\left(t_{i}\right)\right| \leq & \left|\left(T\left(t_{i}-h\right)-T\left(t_{i}\right)\right) \phi(0)\right| \\
& +\int_{0}^{t_{i}-h}\left|\left(T\left(t_{i}-h-s\right)-T\left(t_{i}-s\right)\right) f\left(s, y_{s}\right)\right| d s \\
& +\psi(q) M \int_{t_{i}-h}^{t_{i}} p(s) d s
\end{aligned}
$$

which tends to zero as $h \rightarrow 0$.

Define

$$
\widehat{\mathcal{B}}_{0}(y)(t)=\mathcal{B}(y)(t), \quad t \in\left[0, t_{1}\right]
$$

and

$$
\widehat{\mathcal{B}}_{i}(y)(t)=\left\{\begin{array}{cc}
\mathcal{B}(y)(t), & \text { if } t \in\left(t_{i}, t_{i+1}\right] \\
\mathcal{B}(y)\left(t_{i}^{+}\right), & \text {if } t=t_{i} .
\end{array}\right.
$$

Next we prove equicontinuity at $t=t_{i}^{+}$. Fix $\delta_{2}>0$ such that $\left\{t_{k}: k \neq i\right\} \cap\left[t_{i}-\delta_{2}, t_{i}+\delta_{2}\right]=\emptyset$. For $0<h<\delta_{2}$ we have

$$
\begin{aligned}
\left|\widehat{\mathcal{B}}(y)\left(t_{i}+h\right)-\widehat{\mathcal{B}}(y)\left(t_{i}\right)\right| \leq \quad & \left|\left(T\left(t_{i}+h\right)-T\left(t_{i}\right)\right) \phi(0)\right| \\
& +\int_{0}^{t_{i}}\left|\left(T\left(t_{i}+h-s\right)-T\left(t_{i}-s\right)\right) f\left(s, y_{s}\right)\right| d s \\
& +\psi(q) M \int_{t_{i}}^{t_{i}+h} p(s) d s .
\end{aligned}
$$


The right-hand side tends to zero as $h \rightarrow 0$. The equicontnuity for the cases $\tau_{1}<\tau_{2} \leq 0$ and $\tau_{1} \leq 0 \leq \tau_{2}$ follows from the uniform continuity of $\phi$ on the interval $[-r, 0]$.

As consequence of Steps 1 to 3 together with Arzelá-Ascoli theorem it suffices to show that $\mathcal{B}$ maps $B$ into a precompact set in $E$.

Let $0<t<b$ be fixed and let $\epsilon$ be a real number satisfying $0<\epsilon<t$. For $y \in B_{q}$ we define

$$
\mathcal{B}_{\epsilon}(y)(t)=T(t) \phi(0)+T(\epsilon) \int_{0}^{t-\epsilon} T(t-s-\epsilon) f\left(s, y_{s}\right) d s .
$$

Since $T(t)$ is a compact operator, the set

$$
Y_{\epsilon}(t)=\left\{\mathcal{B}_{\epsilon}(y)(t): \quad y \in B_{q}\right\}
$$

is precompact in $E$ for every $\epsilon, 0<\epsilon<t$. Moreover, for every $y \in B_{q}$ we have

$$
\begin{aligned}
\left|\mathcal{B}(y)(t)-\mathcal{B}_{\epsilon}(y)(t)\right| & \leq \psi(q) \int_{t-\epsilon}^{t}\|T(t-s)\|_{B(E)} p(s) d s \\
& \leq \psi(q) M \int_{t-\epsilon}^{t} p(s) d s .
\end{aligned}
$$

Therefore, there are precompact sets arbitrarily close to the set $Y_{\epsilon}(t)=\left\{\mathcal{B}_{\epsilon}(y)(t): \quad y \in B_{q}\right\}$. Hence the set $Y(t)=\left\{\mathcal{B}(y)(t): \quad y \in B_{q}\right\}$ is precompact in $E$. Hence the operator $\mathcal{B}: P C([-r, b], E) \rightarrow$ $P C([-r, b], E)$ is completely continuous.

Step 4: $\mathcal{A}$ is a contraction

Let $x, y \in P C([-r, b], E)$. Then for $t \in J$

$$
\begin{aligned}
|\mathcal{A}(y)(t)-\mathcal{A}(x)(t)| & =\left|\sum_{0<t_{k}<t} T\left(t-t_{k}\right)\left(I_{k}\left(y\left(t_{k}^{-}\right)\right)-I_{k}\left(x\left(t_{k}^{-}\right)\right)\right)\right| \\
& \leq M \sum_{0<t_{k}<t}\left|I_{k}\left(y\left(t_{k}^{-}\right)\right)-I_{k}\left(x\left(t_{k}^{-}\right)\right)\right| \\
& \leq M \sum_{k=1}^{m} d_{k}\left|y\left(t_{k}^{-}\right)-x\left(t_{k}^{-}\right)\right| \\
& \leq M \sum_{k=1}^{m} d_{k}\|y-x\| .
\end{aligned}
$$

Then

$$
\|\mathcal{A}(y)-\mathcal{A}(x)\| \leq M \sum_{k=1}^{m} d_{k}\|y-x\|
$$

which is a contraction, since $M \sum_{k=1}^{m} d_{k}<1$.

Step 5: A priori bounds. 
Now it remains to show that the set

$$
\mathcal{E}=\left\{y \in P C([-r, b], E): y=\lambda \mathcal{B}(y)+\lambda \mathcal{A}\left(\frac{y}{\lambda}\right) \text { for some } 0<\lambda<1\right\}
$$

is bounded.

Let $y \in \mathcal{E}$, then $y=\lambda \mathcal{B}(y)+\lambda \mathcal{A}\left(\frac{y}{\lambda}\right)$ for some $0<\lambda<1$. Thus, for each $t \in J$,

$$
y(t)=\lambda T(t) \phi(0)+\lambda \int_{0}^{t} T(t-s) f\left(s, y_{s}\right) d s+\lambda \sum_{0<t_{k}<t} T\left(t-t_{k}\right) I_{k}\left(\frac{y}{\lambda}\left(t_{k}^{-}\right)\right) .
$$

This implies by (H2) and (H4) that, for each $t \in J$, we have

$$
\begin{aligned}
|y(t)| \leq & \lambda M|\phi(0)|+\lambda M \int_{0}^{t} p(s) \psi\left(\left\|y_{s}\right\|\right) d s+\lambda M \sum_{k=1}^{m}\left|I_{k}\left(\frac{y}{\lambda}\left(t_{k}^{-}\right)\right)\right| \\
\leq & \lambda M\|\phi\|+\lambda M \int_{0}^{t} p(s) \psi\left(\left\|y_{s}\right\|\right) d s \\
& +\lambda M \sum_{k=1}^{m}\left|I_{k}\left(\frac{y}{\lambda}\left(t_{k}^{-}\right)\right)-I_{k}(0)\right|+\lambda M \sum_{k=1}^{m}\left|I_{k}(0)\right| \\
\leq & \lambda M\left(\|\phi\|+\sum_{k=1}^{m}\left|I_{k}(0)\right|\right)+\lambda M \int_{0}^{t} p(s) \psi\left(\left\|y_{s}\right\|\right) d s \\
& +\lambda M \sum_{k=1}^{m} d_{k}\left|\frac{y}{\lambda}\left(t_{k}^{-}\right)\right| \\
\leq & M\left(\|\phi\|+\sum_{k=1}^{m}\left|I_{k}(0)\right|\right)+M\left[\int_{0}^{t} p(s) \psi\left(\left\|y_{s}\right\|\right) d s+\sum_{k=1}^{m} d_{k}\left|y\left(t_{k}^{-}\right)\right|\right] .
\end{aligned}
$$

Now we consider the function $\mu$ defined by

$$
\mu(t)=\sup \{|y(s)|: \quad-r \leq s \leq t\}, \quad 0 \leq t \leq b .
$$

Then $\left\|y_{s}\right\| \leq \mu(t)$ for all $t \in J$ and there is a point $t^{*} \in[-r, t]$ such that $\mu(t)=\left|y\left(t^{*}\right)\right|$. If $t^{*} \in[0, b]$, by the previous inequality we have for $t \in[0, b]$ (note $t^{*} \leq t$ )

$$
\mu(t) \leq M\left(\|\phi\|+\sum_{k=1}^{m}\left|I_{k}(0)\right|\right)+M \int_{0}^{t} p(s) \psi(\mu(s)) d s+M \sum_{k=1}^{m} d_{k} \mu(t) .
$$

Then

$$
\left(1-M \sum_{k=1}^{m} d_{k}\right) \mu(t) \leq M\left(\|\phi\|+\sum_{k=1}^{m}\left|I_{k}(0)\right|\right)+M \int_{0}^{t} p(s) \psi(\mu(s)) d s .
$$


Thus we have

$$
\mu(t) \leq D_{0}+D_{1} \int_{0}^{t} p(s) \psi(\mu(s)) d s .
$$

Let us take the right hand-side of above inequality as $v(t)$. Then we have

$$
\begin{gathered}
\mu(t) \leq v(t) \text { for all } t \in J, \\
v(0)=D_{0},
\end{gathered}
$$

and

$$
v^{\prime}(t)=D_{1} p(t) \psi(\mu(t)), \text { a.e. } t \in J .
$$

Using the nondecreasing character of $\psi$ we get

$$
v^{\prime}(t) \leq D_{1} p(t) \psi(v(t)), \text { a.e. } t \in J .
$$

That is

$$
\frac{v^{\prime}(t)}{\psi(v(t))} \leq D_{1} p(t), \text { a.e. } t \in J
$$

Integrating from 0 to $t$ we get

$$
\int_{0}^{t} \frac{v^{\prime}(s)}{\psi(v(s))} d s \leq D_{1} \int_{0}^{t} p(s) d s
$$

By a change of variable we get

$$
\int_{v(0)}^{v(t)} \frac{d u}{\psi(u)} \leq D_{1} \int_{0}^{b} p(s) d s=D_{1}\|p\|_{L^{1}}<\int_{D_{0}}^{\infty} \frac{d u}{\psi(u)} .
$$

Hence there exist a constant $N$ such that

$$
\mu(t) \leq v(t) \leq N \text { for all } t \in J .
$$

Now from the definition of $\mu$ it follows that

$$
\|y\|=\sup _{t \in[-r, b]}|y(t)| \leq \mu(b) \leq N, \quad \text { for all } y \in \mathcal{E} .
$$

This shows that the set $\mathcal{E}$ is bounded. As a consequence of Theorem 3.1 we deduce that $\mathcal{A}+\mathcal{B}$ has a fixed point which is a mild solution of problem (1)-(3).

\section{Existence of Extremal Mild Solutions}

In this section we shall prove the existence of maximal and minimal solutions of problem (1)-(3) under suitable monotonicity conditions on the functions involved in it.

Definition 4.1. A nonempty closed subset $C$ of a Banach space $(X,\|\cdot\|)$ is said to be a cone if 
(i) $C+C \subset C$,

(ii) $\lambda C \subset C$

(iii) $\{-C\} \cap\{C\}=\{0\}$

A cone $C$ is called normal if the norm $\|\cdot\|$ is semi-monotone on $C$, i.e., there exists a constant $N>0$ such that $\|x\| \leq N\|y\|$, whenever $x \leq y$. We equip the space $X=C(J, E)$ with the order relation $\leq$ induced by a regular cone $\mathcal{C}$ in $E$, that is for all $y, \bar{y} \in X: y \leq \bar{y}$ if and only if $\bar{y}(t)-y(t) \in \mathcal{C}, \forall t \in J$. In what follows will assume that the cone $C$ is normal. Cones and their properties are detailed in $[17,20]$. Let $a, b \in X$ be such that $a \leq b$. Then, by an order interval $[a, b]$ we mean a set of points in $X$ given by

$$
[a, b]=\{x \in X \mid a \leq x \leq b\} .
$$

Definition 4.2. Let $X$ be an ordered Banach space. A mapping $T: X \rightarrow X$ is called isotone increasing if $T(x) \leq T(y)$ for any $x, y \in X$ with $x<y$. Similarly, $T$ is called isotone decreasing if $T(x) \geq T(y)$ whenever $x<y$.

Definition 4.3. We say that $x \in X$ is the least fixed point of $G$ in $X$ if $x=G x$ and $x \leq y$ whenever $y \in X$ and $y=G y$. The greatest fixed point of $G$ in $X$ is defined similarly by reversing the inequality. If both least and greatest fixed point of $G$ in $X$ exist, we call them extremal fixed point of $G$ in $X$.

Very recently Dhage has proved the following.

Theorem 4.1. [16]. Let $[a, b]$ be an order interval in a Banach space $X$ and let $B_{1}, B_{2}:[a, b] \rightarrow X$ be two functions satisfying:

(a) $B_{1}$ is a contraction,

(b) $B_{2}$ is completely continuous,

(c) $B_{1}$ and $B_{2}$ are strictly monotone increasing, and

(d) $B_{1}(x)+B_{2}(x) \in[a, b], \forall x \in[a, b]$.

Further if the cone $C$ in $X$ is normal, then the equation $x=B_{1}(x)+B_{2}(x)$ has a least fixed point $x_{*}$ and a greatest fixed point $x^{*} \in[a, b]$. Moreover $x_{*}=\lim _{n \rightarrow \infty} x_{n}$ and $x^{*}=\lim _{n \rightarrow \infty} y_{n}$, where $\left\{x_{n}\right\}$ and $\left\{y_{n}\right\}$ are the sequences in $[a, b]$ defined by

$$
x_{n+1}=B_{1}\left(x_{n}\right)+B_{2}\left(x_{n}\right), x_{0}=a \text { and } y_{n+1}=B_{1}\left(y_{n}\right)+B_{2}\left(y_{n}\right), y_{0}=b .
$$

We need the following definitions in the sequel.

Definition 4.4. We say that a function $v \in P C([-r, b], E)$ is a lower mild solution of problem (1)-(3) if $v(t)=\phi(t), t \in[-r, 0]$, and

$$
v(t) \leq T(t) \phi(0)+\int_{0}^{t} T(t-s) f\left(s, v_{s}\right) d s+\sum_{0<t_{k}<t} T\left(t-t_{k}\right) I_{k}\left(v\left(t_{k}^{-}\right)\right), t \in J, t \neq t_{k}
$$

and $v\left(t_{k}^{+}\right)-v\left(t_{k}^{-}\right) \leq I_{k}\left(v\left(t_{k}\right)\right), t=t_{k}, k=1, \ldots, m$. Similarly an upper mild solution $w$ of problem (1)-(3) is defined by reversing the order. 
Definition 4.5. A solution $x_{M}$ of problem (1)-(3) is said to be maximal if for any other solution $x$ of problem (1)-(3) on $J$, we have that $x(t) \leq x_{M}(t)$ for each $t \in J$.

Similarly a minimal solution of problem (1)-(3) is defined by reversing the order of the inequalities.

Definition 4.6. A function $f(t, x)$ is called strictly monotone increasing in $x$ almost everywhere for $t \in J$, if $f(t, x) \leq f(t, y)$ a.e. $t \in J$ for all $x, y \in D$ with $x<y$. Similarly $f(t, x)$ is called strictly monotone decreasing in $x$ almost everywhere for $t \in J$, if $f(t, x) \geq f(t, y)$ a.e. $t \in J$ for all $x, y \in D$ with $x<y$.

We consider the following assumptions in the sequel.

(H5) The function $f(t, y)$ is strictly monotone increasing in $y$ for almost each $t \in J$.

(H6) $T(t)$ is preserving the order, that is $T(t) v \geq 0$ whenever $v \geq 0$.

(H7) The function $I_{k}, k=1, \ldots m$ are continuous and nondecreasing.

(H8) The problem (1)-(3) has a lower mild solution $v$ and an upper mild solution $w$ with $v \leq w$.

Theorem 4.2. Assume that assumptions (H1)-(H8) hold. Then problem (1)-(3) has minimal and maximal solutions on $[-r, b]$.

Proof. It can be shown, as in the proof of Theorem 3.2 that $\mathcal{B}$ is completely continuous and $\mathcal{A}$ is a contraction on $[v, w]$. We shall show that $\mathcal{A}$ and $\mathcal{B}$ are isotone increasing on $[v, w]$. Let $y, \bar{y} \in[a, b]$ be such that $y \leq \bar{y}, y \neq \bar{y}$. Then by (H5), (H6), we have for each $t \in J$

$$
\begin{aligned}
\mathcal{B}(y)(t) & =T(t) \phi(0)+\int_{0}^{t} T(t-s) f\left(s, y_{s}\right) d s \\
& \leq T(t) \phi(0)+\int_{0}^{t} T(t-s) f\left(s, \bar{y}_{s}\right) d s \\
& =\mathcal{B}(\bar{y})(t) .
\end{aligned}
$$

and by (H7), we have for each $t \in J$

$$
\begin{aligned}
\mathcal{A}(y)(t) & =\sum_{0<t_{k}<t} T\left(t-t_{k}\right) I_{k}\left(y\left(t_{k}^{-}\right)\right) \\
& \leq \sum_{0<t_{k}<t} T\left(t-t_{k}\right) I_{k}\left(\bar{y}\left(t_{k}^{-}\right)\right) \\
& =\mathcal{A}(\bar{y})(t) .
\end{aligned}
$$

Therefore $\mathcal{A}$ and $\mathcal{B}$ are isotone increasing on $[v, w]$. Finally, let $x \in[v, w]$ be any element. By (H8) we deduce that

$$
v \leq \mathcal{A}(v)+\mathcal{B}(v) \leq \mathcal{A}(x)+\mathcal{B}(x) \leq \mathcal{A}(w)+\mathcal{B}(w) \leq w,
$$

which shows that $\mathcal{A}(x)+\mathcal{B}(x) \in[v, w]$ for all $x \in[v, w]$. Thus, $\mathcal{A}$ and $\mathcal{B}$ satisfy all conditions of Theorem 4.1 , hence problem (1)-(3) has maximal and minimal solutions on $[-r, b]$. This completes the proof. 


\section{Impulsive Functional Differential Equations with Non-local Conditions}

In this section we shall prove existence results for problem (4)-(6). Nonlocal conditions were initiated by Byszewski [11] when he proved the existence and uniqueness of mild and classical solutions of nonlocal Cauchy problems. As remarked by Byszewski [12], the nonlocal condition can be more useful than the standard initial condition to describe some physical phenomena.

Definition 5.1. A function $y \in P C([-r, b], E)$ is said to be a mild solution of problem (4)-(6) if $y(t)=\phi(t)-h_{t}(y), t \in[-r, 0]$, and

$$
y(t)=T(t)\left(\phi(0)-h_{0}(y)\right)+\int_{0}^{t} T(t-s) f\left(s, y_{s}\right) d s+\sum_{0<t_{k}<t} T\left(t-t_{k}\right) I_{k}\left(y\left(t_{k}^{-}\right)\right), \quad t \in J .
$$

Theorem 5.1. Assume that hypotheses (H1)-(H3) hold and moreover

(A1) The function $h$ is continuous with respect to $t$, and there exists a constant $\alpha>0$ such that

$$
\left|h_{t}(u)\right| \leq \alpha, \quad u \in P C([-r, b], E)
$$

and for each $k>0$ the set

$$
\left\{\phi(0)-h_{0}(y), y \in P C([-r, b], E),\|y\| \leq k\right\}
$$

is precompact in $E$

(A2) There exists a function $p \in L^{1}\left(J, \mathbb{R}_{+}\right)$and a continuous nondecreasing function $\psi:[0, \infty) \rightarrow$ $(0, \infty)$ such that

$$
|f(t, x)| \leq p(t) \psi\left(\|x\|_{D}\right), \quad \text { a.e. } t \in J, \quad \text { for all } x \in D
$$

with

$$
\int_{\tilde{D}_{0}}^{\infty} \frac{d s}{\psi(s)}>D_{1}\|p\|_{L^{1}}
$$

and

$$
\tilde{D}_{0}=\frac{M\left[\|\phi\|_{D}+\alpha+\sum_{k=1}^{m}\left|I_{k}(0)\right|\right]}{1-M \sum_{k=1}^{m} d_{k}} .
$$

Then the problem 4)-(6) has at least one mild solution on $[-r, b]$.

Proof. Transform the problem (4)-(6) into a fixed point problem. Consider the two operators : $\mathcal{B}_{1}: P C([-r, b], E) \rightarrow P C([-r, b], E)$ defined by

$$
\mathcal{B}_{1}(y)(t)= \begin{cases}\phi(t)-h_{t}(y), & \text { if } t \in[-r, 0] ; \\ T(t)\left(\phi(0)-h_{0}(y)\right)+\int_{0}^{t} T(t-s) f\left(s, y_{s}\right) d s, & \text { if } t \in J,\end{cases}
$$


and

$$
\mathcal{A}_{1}(y)(t)= \begin{cases}0, & \text { if } t \in[-r, 0] ; \\ \sum_{0<t_{k}<t} T\left(t-t_{k}\right) I_{k}\left(y\left(t_{k}^{-}\right)\right), & \text {if } t \in J .\end{cases}
$$

Then the problem of finding the solution of problem (4)-(6) is reduced to finding the solution of the operator equation $\mathcal{A}_{1}(y)(t)+\mathcal{B}_{2}(y)(t)=y(t), t \in[-r, b]$. As in Section 3, we can show that the operators $\mathcal{A}_{1}$ and $\mathcal{B}_{1}$ satisfy all conditions of Theorem 3.1.

\section{An Example}

As an application of our results we consider the following impulsive partial functional differential equation of the form

$$
\begin{gathered}
\frac{\partial}{\partial t} z(t, x)=\frac{\partial^{2}}{\partial x^{2}} z(t, x) \\
+Q(t, z(t-r, x)), x \in[0, \pi], t \in[0, b] \backslash\left\{t_{1}, t_{2}, \ldots, t_{m}\right\} . \\
z\left(t_{k}^{+}, x\right)-z\left(t_{k}^{-}, x\right)=b_{k} z\left(t_{k}^{-}, x\right), x \in[0, \pi], k=1, \ldots, m \\
z(t, 0)=z(t, \pi)=0, t \in[0, b] \\
z(t, x)=\phi(t, x), t \in[-r, 0], x \in[0, \pi],
\end{gathered}
$$

where $r>0, b_{k}>0, k=1, \ldots, m, \phi \in \mathcal{D}=\{\psi:[-r, 0] \times[0, \pi] \rightarrow \mathbb{R} ; \psi$ is continuous everywhere except for a countable number of points at which $\psi\left(s^{-}\right), \psi\left(s^{+}\right)$exist with $\left.\psi\left(s^{-}\right)=\psi(s)\right\}, 0=t_{0}<$ $t_{1}<t_{2}<\ldots<t_{m}<t_{m+1}=b, z\left(t_{k}^{+}\right)=\lim _{(h, x) \rightarrow\left(0^{+}, x\right)} z\left(t_{k}+h, x\right), z\left(t_{k}^{-}\right)=\lim _{(h, x) \rightarrow\left(0^{-}, x\right)} z\left(t_{k}+h, x\right)$ and $Q:[0, b] \times \mathbb{R} \rightarrow \mathbb{R}$ is a given function.

Let

$$
\begin{gathered}
y(t)(x)=z(t, x), t \in J, x \in[0, \pi], \\
I_{k}\left(y\left(t_{k}^{-}\right)\right)(x)=b_{k} z\left(t_{k}^{-}, x\right), x \in[0, \pi], k=1, \ldots, m \\
F(t, \phi)(x)=Q(t, \phi(\theta, x)), \theta \in[-r, 0], x \in[0, \pi], \\
\phi(\theta)(x)=\phi(\theta, x), \theta \in[-r, 0], x \in[0, \pi] .
\end{gathered}
$$

Take $E=L^{2}[0, \pi]$ and define $A: D(A) \subset E \rightarrow E$ by $A w=w^{\prime \prime}$ with domain

$$
D(A)=\left\{w \in E, w, w^{\prime} \quad \text { are absolutely continuous, } w^{\prime \prime} \in E, w(0)=w(\pi)=0\right\} .
$$

Then

$$
A w=\sum_{n=1}^{\infty} n^{2}\left(w, w_{n}\right) w_{n}, w \in D(A)
$$

where $(, \quad)$ is the inner product in $L^{2}$ and $w_{n}(s)=\sqrt{\frac{2}{\pi}} \sin n s, n=1,2, \ldots$ is the orthogonal set of eigenvectors in $A$. It is well known (see [26]) that $A$ is the infinitesimal generator of an analytic semigroup $T(t), t \in[0, b]$ in $E$ and is given by

$$
T(t) w=\sum_{n=1}^{\infty} \exp \left(-n^{2} t\right)\left(w, w_{n}\right) w_{n}, w \in E .
$$


Since the analytic semigroup $T(t)$ is compact, there exists a constant $M \geq 1$ such that

$$
\|T(t)\|_{B(E)} \leq M
$$

Also assume that there exists an integrable function $\sigma:[0, b] \rightarrow \mathbb{R}^{+}$such that

$$
|Q(t, w(t-r, x))| \leq \sigma(t) \Omega(|w|)
$$

where $\Omega:[0, \infty) \rightarrow(0, \infty)$ is continuous and nondecreasing with

$$
\int_{1}^{\infty} \frac{d s}{s+\Omega(s)}=+\infty
$$

Assume that there exists a function $\tilde{l} \in L^{1}\left([0, b], \mathbb{R}^{+}\right)$such that

$$
|Q(t, w)-Q(t, \bar{w})| \leq \tilde{l}(t)|w-\bar{w}|, t \in[0, b], w, \bar{w} \in \mathbb{R} .
$$

We can show that problem (1)-(3) is an abstract formulation of problem (7)-(10). Since all the conditions of Theorem 3.2 are satisfied, the problem (7)-(10) has a solution $z$ on $[-r, b] \times[0, \pi]$.

Acknowledgement. This work was completed when the second author was visiting the ICTP in Trieste as a Regular Associate. It is a pleasure for him to express gratitude for its financial support and the warm hospitality.

Received: December 2008. Revised: January 2009.

\section{References}

[1] N. U. Ahmed, Semigroup Theory with Applications to Systems and Control, Pitman Research Notes in Mathematics Series, 246. Longman Scientific \& Technical, Harlow; John Wiley \& Sons, New York, 1991.

[2] N. U. Ahmed, Dynamic Systems and Control with Applications, World Scientific Publishing Co. Pte. Ltd., Hackensack, NJ, 2006.

[3] N. U. Ahmed, Systems governed by impulsive differential inclusions on Hilbert spaces, Nonlinear Anal. 45 (2001), 693-706.

[4] N. U. Ahmed, Optimal control for impulsive systems in Banach spaces, Inter. J. Differ. Equ. Appl. 1 (1) (2000), 37-52.

[5] D.D. Bainov And P.S. Simeonov, Systems with Impulsive effect, Horwood, Chichister, 1989.

[6] M. Benchohra, J. Henderson and S. K. Ntouyas, Impulsive Differential Equations and Inclusions, Hindawi Publishing Corporation, Vol 2, New York, 2006.

[7] M. Benchohra and S.K. Ntouyas, Existence and controllability results for multivalued semilinear differential equations with nonlocal conditions, Soochow J. Math. 29 (2003),157-170. 
[8] M. Benchohra and S. K. Ntouyas, Existence of mild solutions for certain delay semilinear evolution inclusions with nonlocal condition, Dynam. Systems Appl. 9 (3) (2000), 405-412.

[9] M. Benchohra and S. K. Ntouyas, Existence of mild solutions of semilinear evolution inclusions with nonlocal conditions, Georgian Math. J. 7 (2) (2002), 221 - 230.

[10] T.A. Burton And C. Kirk, A fixed point theorem of Krasnoselskiii-Schaefer type, Math. Nachr. 189 (1998), 23-31.

[11] L. Byszewski, Theorems about existence and uniqueness of solutions of a semilinear evolution nonlocal Cauchy problem, J. Math. Anal. Appl. 162 (1991), 494-505.

[12] L. Byszewski, Existence and uniqueness of mild and classical solutions of semilinear functionaldifferential evolution nonlocal Cauchy problem. Selected problems of mathematics, 25-33, 50th Anniv. Cracow Univ. Technol. Anniv. Issue, 6, Cracow Univ. Technol., Krakow, 1995

[13] L. Byszewski And H. AkCA, On a mild solution of a semilinear functional-differential evolution nonlocal problem, J. Appl. Math. Stochastic Anal. 10 (1997), 265-271.

[14] L. Byszewski And V. Lakshmikantham, Theorem about the existence and uniqueness of a solution of a nonlocal abstract Cauchy problem in a Banach space, Appl. Anal. 40 (1991), 11-19.

[15] T. Cardinali and P. Rubbioni, Mild solutions for impulsive semilinear evolution differential inclusions. J. Appl. Funct. Anal. 1 (2006), 303-325.

[16] B. C. DhAGE, Fixed-point theorems for discontinuous multivalued operators on ordered spaces with applications, Comput. Math. Appl. 51 (2006), 589-604.

[17] D. Guo and V. Lakshmikantham, Nonlinear Problems in Abstract Cones, Academic Press, New York, 1988.

[18] J. K. Hale, Theory of Functional Differential Equations, Springer-Verlag, New York, 1977.

[19] J. K. Hale And S. Verduyn Lunel, Introduction to Functional -Differential Equations, Applied Mathematical Sciences, 99, Springer-Verlag, New York, 1993.

[20] S. Heikkila and V. Lakshmikantham, Monotone Iterative Technique for Nonlinear Discontinuous Differential Equations, Marcel Dekker Inc., New York, 1994.

[21] Sh. Hu and N. Papageorgiou, Handbook of Multivalued Analysis, Volume I: Theory, Kluwer Academic Publishers, Dordrecht, 1997.

[22] M. Kamenskit, V. Obukhovskir and P. Zecca, Condensing Multivalued Maps and Semilinear Differential Inclusions in Banach Spaces, de Gruyter Series in Nonlinear Analysis and Applications, Berlin, 2001.

[23] V. Kolmanovskit, And A. Myshkis, Introduction to the Theory and Applications of Functional-Differential Equations. Mathematics and its Applications, 463. Kluwer Academic Publishers, Dordrecht, 1999. 
[24] V. Lakshmikantham, D.D. Bainov and P.S. Simeonov, Theory of Impulsive Differntial Equations, Worlds Scientific, Singapore, 1989.

[25] J.H. Liu, Nonlinear impulsive evolution equations, Dynam. Contin. Discrete Impuls. Systems 6 (1999), 77-85.

[26] A. PAZY, Semigroups of Linear Operators and Applications to Partial Differential Equations, Springer-Verlag, New York, 1983.

[27] Yuri V. Rogovchenko, Impulsive evolution systems: Main results and new trends, Dyn. Contin. Discrete Impuls. Syst. 3 (1) (1997), 57-88.

[28] Yuri V. Rogovchenko, Nonlinear impulsive evolution systems and applications to population models, J. Math. Anal. Appl. 207 (2) (1997), 300-315.

[29] A.M. Samoilenko and N.A. Perestyuk, Impulsive Differential Equations World Scientific, Singapore, 1995.

[30] J. Wu, Theory and Applications of Partial Functional Differential Equations, Applied Mathematical Sciences 119, Springer-Verlag, New York, 1996. 